\title{
An Efficient Numerical Interface Between FDTD and Haar MRTD_Formulation and Applications
}

\author{
Costas D. Sarris, Member, IEEE, and Linda P. B. Katehi, Fellow, IEEE
}

\begin{abstract}
A hybrid finite-difference time-domain (FDTD)/Haar multiresolution time-domain (MRTD) technique for the time-domain analysis of microwave structures is proposed in this paper. The salient features of the presented algorithm are, first, its inherent stability that stems from the matching of the dispersion properties of FDTD and Haar MRTD and, second, its applicability to arbitrarily high wavelet order MRTD schemes. Thus, the application of the MRTD technique to the modeling of open structures and inhomogeneous circuit geometries is facilitated. In particular, the straightforward implementation of perfectly matched layer type and Mur's absorbing boundary conditions is attained. The fact that the proposed interface involves no spatial or temporal interpolations or extrapolations indicates its potential to efficiently connect FDTD and Haar MRTD.
\end{abstract}

Index Terms-Finite-difference time-domain (FDTD), multiresolution analysis, multiresolution time-domain (MRTD).

\section{INTRODUCTION}

$\mathbf{T}$ HE time-domain characterization of microwave structures, often encountered in wireless front-end applications such as filter, resonator, or feed components, usually includes the modeling of fine detail complex boundaries and regions of dynamically varying field distributions [1]. For this purpose, dense gridding conditions are necessary for the extraction of an accurate solution to the problem at hand. Nevertheless, the implementation of a uniformly dense mesh for an electrically large structure translates to computationally burdensome simulations, as a result of both the size of the domain (in cells) and the stability-related restriction on the time step of a finite-difference time-domain (FDTD) type of scheme, imposed by the small dimensions of the unit cell. Hence, the incorporation of local mesh refinement techniques into conventional time-domain solvers is motivated as a means of alleviating the overall cost of modeling structures with localized geometric details.

Standard subgridding techniques involve spatio-temporal interpolations or extrapolations at the boundaries of different resolution parts of the numerical grid [2], [3] that render the rig-

Manuscript received March 3, 2002; revised December 2, 2002. This work was supported by the U.S. Army Communications and Electronics Command and by the Department of Defense High-Performance Computing Modernization Program under the "Efficient Numerical Solutions to Large Scale Tactical Communication Problems" Project DAAD19-00-1-0173.

C. D. Sarris was with the Radiation Laboratory, Department of Electrical Engineering and Computer Science, University of Michigan at Ann Arbor, Ann Arbor, MI 48109-2122 USA. He is now with the E. S. Rogers Sr. Department of Electrical and Computer Engineering, University of Toronto, Toronto, ON, Canada M5S 3G4.

L. P. B. Katehi was with the Radiation Laboratory, Department of Electrical Engineering and Computer Science, University of Michigan at Ann Arbor, Ann Arbor, MI 48109-2122 USA. She is now with the Department of Electrical and Computer Engineering, Purdue University, West Lafayette, IN 47097 USA. Digital Object Identifier 10.1109/TMTT.2003.809620

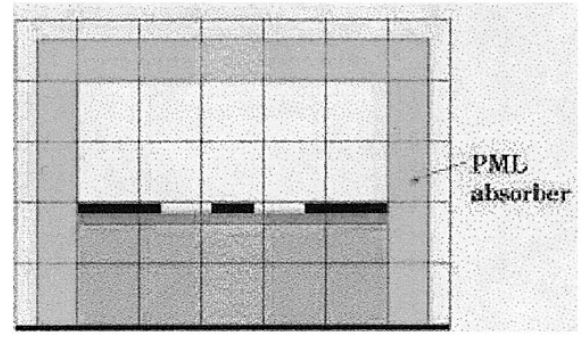

Fig. 1. Coarse rectangular mesh for a layered coplanar-waveguide structure with cells containing variable dielectric permittivity and electric conductivity profiles.

orous enforcement of the divergence-free nature of the magnetic field and the continuity conditions a rather subtle issue. Furthermore, a higher order static subgridded FDTD algorithm that needs no interpolatory operations has been recently proposed in [4]. However, as time-domain simulations of microwave geometries typically register the history of a wide-band pulse propagation along a computational domain, adaptively imposing dense gridding conditions only in and around the pulse and the products of its retro-reflections can further extend the efficiency of a technique. Thus, if allowing for a relatively coarse mesh is one challenge that novel numerical schemes are expected to meet, a second, but equally important one, is adaptivity, translating to the possibility of dynamic mesh refinement at regions of the domain that are electromagnetically active at a certain time step.

Wavelet-based numerical algorithms stemming from the multiresolution time-domain (MRTD) technique [5] offer a natural framework for the implementation of dynamic mesh refinement in the sense demonstrated in [6]. For homogeneous domains, the computational efficiency of the former is expected to increase with the order of the multiresolution expansion. Yet, the complexity of conductor, dielectric, and boundary modeling that they present, also increasing with wavelet order, seriously compromises their potential applicability to state-of-the-art devices. In particular, whenever a single cell includes variable material properties (such as dielectric and/or conducting layers), direct MRTD update equations are replaced by matrix expressions, resulting from the discretization of constitutive relations [5], [13]. These cases are encountered in typical microwave devices such as microstrip lines and coplanar waveguides (Fig. 1). In addition, the most effective mesh truncation technique today, the perfectly matched layer (PML) absorber, is itself an inhomogeneous uniaxially anisotropic (both electrically and magnetically) material.

The aforementioned contradiction was addressed by the authors in [7] and [8] by means of a hybrid approach that connected the FDTD technique with the Haar wavelet-based MRTD via a numerical interface. The purpose of this approach was to 
establish an efficient algorithm, allowing for the combination of the versatility of FDTD with the adaptivity of MRTD, employing the first in geometrically complex parts of the domain and the second in homogeneous regions. This paper presents the ideas of [8] in greater extent.

In the past, interfaces between different numerical methods have been developed in order to address specific problems of interest. In [9], the frequency-domain method of moments (MoM) was coupled to the FDTD technique for the analysis of ground penetrating radar (GPR) problems, where GPR antenna operation was modeled by the MoM, while the inhomogeneous ground (including dielectric stratification) was incorporated in an FDTD mesh. In [10], a Daubechies scaling-function-based waveletGalerkin scheme was coupled to FDTD for the modeling of wedge loaded two-dimensional waveguide structures, apparently leading to significant computational savings compared to its pure FDTD counterpart. Still, the general applicability and efficiency of the scheme was questionable, given the largely different dispersion characteristics of the two methods. Moreover, a hybridization of the transmission line matrix (TLM) method and MRTD was pursued in [11], where the issue of dealing with disparate dispersion methods was explicitly addressed via a conditionally stable space-time interpolation scheme. Finally, [12] demonstrated a technique for including FDTD-modeled lumped elements in a three-dimensional domain simulated by the Haar wavelet-based MRTD formulation that was introduced in [13] and was restricted to one wavelet level.

This paper proposes an interface between a Haar wavelet MRTD scheme of an arbitrary number of wavelet levels and FDTD technique. Under certain conditions, which are mentioned in [14], the two schemes are characterized by the same dispersion properties; a fact that is utilized in order to couple them with no interpolations or extrapolations and with absolutely no spurious reflections at their interface. This paper is organized as follows. First, the formulation of an arbitraryorder Haar MRTD scheme is presented. Noting that the dispersion equation for the latter coincides with the dispersion equation of an FDTD scheme of the same spatial resolution, a simple connection algorithm between the two is proposed. Emphasis is placed on the implementation problems that arise in the application of update equations at the boundaries of the two domains. Validation studies include the analysis of twodimensional dielectric cavity structures under various FDTD/ MRTD mesh configurations. Finally, the method is applied for mesh truncation in open-domain problems and the modeling of structures with metal inserts such as a fin-loaded cavity.

\section{TWO-DIMENSIONAL HYBRID ARBITRARY ORDER HAAR MRTD/FDTD SCHEME: FORMULATION}

\section{A. MRTD, FDTD Equations}

A two-dimensional system of the following Maxwell's equations corresponding to $\mathrm{TE}_{z}$ waves:

$$
\begin{aligned}
\frac{\partial E_{y}}{\partial t}(\bar{\rho}, t) & =\frac{1}{\epsilon}\left(\frac{\partial H_{x}}{\partial z}(\bar{\rho}, t)-\frac{\partial H_{z}}{\partial x}(\bar{\rho}, t)\right) \\
\frac{\partial H_{x}}{\partial t}(\bar{\rho}, t) & =\frac{1}{\mu} \frac{\partial E_{y}}{\partial z}(\bar{\rho}, t) \\
\frac{\partial H_{z}}{\partial t}(\bar{\rho}, t) & =-\frac{1}{\mu} \frac{\partial E_{y}}{\partial x}(\bar{\rho}, t)
\end{aligned}
$$

with $\bar{\rho}=x \hat{x}+z \hat{z}$ is considered and discretized through the MoM-based technique of [5]. In the MRTD region, the field components are expanded in a Haar wavelet basis of orders $r_{x, \max }$ in the $x$-direction and $r_{z \text {, max }}$ in the $z$-direction, respectively, the scaling cell size being $\Delta x \times \Delta z$. As explained in [14], if an electric field scaling cell is centered at $(i \Delta x, k \Delta z)$, the corresponding $H_{x}$ scaling cell must be centered at $\left(i \Delta x,\left(k+s_{z}\right) \Delta z\right)$, with $s_{z}=1 / 2^{r_{z, \max }+2}$, while the $H_{z}$ one at $\left(\left(i+s_{x}\right) \Delta x, k \Delta z\right)$, with $s_{x}=1 / 2^{r_{x, \max }+2}$. In the FDTD region, the field components are expanded in a pulse basis, introducing the cell dimensions $\Delta \xi_{\text {eff }}=\Delta \xi / 2^{r_{\xi, \max +1}}$, $\xi=x, z$. For example, the $H_{x}$ expansion in the MRTD region is cast in the form

$$
\begin{aligned}
H_{x}(\bar{\rho}, t)=\sum_{n, i, k} & h_{n+(1 / 2)}(t) \\
& \times\left\{n+(1 / 2) H_{i, k+s_{z}}^{x, \phi \phi} \phi_{i}(x) \phi_{k+s_{z}}(z)\right. \\
& +\sum_{r_{x}, p_{x}} n+(1 / 2) H_{i, k+s_{z}}^{x, \psi_{r_{x}} \phi} \psi_{i}^{r_{x}, p_{x}}(x) \phi_{k+s_{z}}(z) \\
& +\sum_{r_{z}, p_{z}} n+(1 / 2) H_{i, k+s_{z}}^{x, \phi \psi_{r_{z}, p_{z}}} \phi_{i}(x) \psi_{k+s_{z}}^{r_{z}, p_{z}}(z) \\
& +\sum_{r_{x}, p_{x}, r_{z}, p_{z}} n+(1 / 2) H_{i, k+s_{z}}^{x, \psi_{x}, p_{x}} \psi_{r_{z}, p_{z}} \\
& \left.\times \psi_{i}^{r_{x}, p_{x}}(x) \psi_{k+s_{z}}^{r_{z}, p_{z}}(z)\right\}
\end{aligned}
$$

where the standard definitions (given, for example, in [14]) have been used for the Haar scaling and wavelet functions $\phi, \psi^{r, p}$, and the pulse functions $h$ (which compose the temporal basis of the method). In the FDTD region, $H_{x}(\bar{\rho}, t)$ is written as

$$
\begin{aligned}
H_{x}(\bar{\rho}, t)= & \sum_{n, i, k} h_{n+(1 / 2)}(t) \\
& \times\left\{{ }_{n+(1 / 2)} H_{i, k+1 / 2} \phi_{i}^{r_{x, \max }+1}(x) \phi_{k+(1 / 2)}^{r_{z, \max }+1}(z)\right\}
\end{aligned}
$$

where the definition of the $r$ th-order scaling function $\phi_{m}^{r}(\xi)=$ $2^{r / 2} \phi\left(2^{r}(\xi / \Delta \xi)-m\right)$ with $\xi=x, z$ has been adopted. The use of Haar wavelets in the MRTD region effectively divides each scaling cell into $2^{r_{x, \max }+1} \times 2^{r_{z, \max }+1}$ subcells of size $\Delta x_{\text {eff }}$ by $\Delta z_{\text {eff }}$, in the sense shown in Fig. 2, where the case of a first-order scheme (with two wavelet levels) in both the $x$ and $z$-direction scheme is depicted. The MRTD equivalent grid points are grid nodes, where field values are computed as linear combinations of the scaling and wavelet terms that appear in (4). A matrix formulation that further illustrates the computation of nodal field values at the equivalent grid points from the coefficients of (4) is provided in [13].

Upon substitution of all field component expansions [such as (4)] into Maxwell's equations (1)-(3), the Galerkin's method is applied for the derivation of field update equations. To this end, Haar integral expressions, which are provided in the Appendix, 


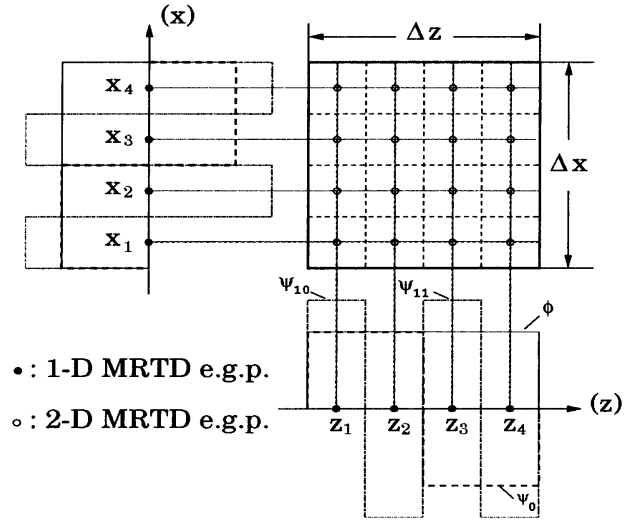

Fig. 2. Equivalent grid points (e.g.p.) within a scaling cell as introduced by a first-order wavelet expansion in the $x$ - and $z$-direction.

are taken into account. In particular, the magnetic-field MRTD coefficients are updated via the following equations:

$$
\begin{aligned}
n^{\prime} H_{i, m^{\prime}}^{x, \chi \phi}= & { }_{n^{\prime}-1} H_{i, m^{\prime}}^{x, \chi \phi}+\frac{\Delta t}{\mu \Delta z} \\
& \times\left\{{ }_{n} E_{i, m+1}^{y, \chi \phi}-{ }_{n} E_{i, m}^{y, \chi \phi}\right. \\
& \left.+\sum_{0 \leq r \leq r_{z, \max }} 2^{r / 2}\left({ }_{n} E_{i, m+1}^{y, \chi \psi_{r, 0}}-{ }_{n} E_{i, m}^{y, \chi \psi_{r, 0}}\right)\right\}
\end{aligned}
$$

$$
\begin{aligned}
& { }_{n^{\prime}} H_{i, m^{\prime}}^{x, \chi \zeta}={ }_{n^{\prime}-1} H_{i, m^{\prime}}^{x, \chi \zeta}+\frac{\Delta t}{\mu \Delta z} \\
& \times\left\{\left({ }_{n} E_{i, m}^{y, \chi \phi}-{ }_{n} E_{i, m+1}^{y, \chi \phi}\right) \times 2^{r_{z} / 2} \delta_{p_{z}, 2^{r z}-1}\right. \\
& +\sum_{r_{z}^{\prime}, p_{z}^{\prime}} \mathcal{D}_{0}\left(r_{z}, p_{z}, r_{z}^{\prime}, p_{z}^{\prime}\right)_{n} E_{i, m+1}^{y, \chi \psi_{r_{z}^{\prime}, p_{z}^{\prime}}} \\
& \left.+\sum_{r_{z}^{\prime}, p_{z}^{\prime}} \mathcal{D}_{1}\left(r_{z}, p_{z}, r_{z}^{\prime}, p_{z}^{\prime}\right)_{n} E_{i, m}^{y, \chi \psi_{r_{z}^{\prime}, p_{z}^{\prime}}}\right\} \\
& { }_{n^{\prime}} H_{i^{\prime}, m}^{z, \phi \eta}={ }_{n^{\prime}-1} H_{i^{\prime}, m}^{z, \phi \eta}-\frac{\Delta t}{\mu \Delta x} \\
& \times\left\{{ }_{n} E_{i+1, m}^{y, \phi \eta}-{ }_{n} E_{i, m}^{y, \phi \eta}\right. \\
& \left.+\sum_{0 \leq r \leq r_{x, \max }} 2^{r / 2}\left({ }_{n} E_{i+1, m}^{y, \psi_{r}, 0} \eta-{ }_{n} E_{i, m}^{y, \psi_{r, 0} \eta}\right)\right\}
\end{aligned}
$$

$$
\begin{aligned}
n^{\prime} H_{i^{\prime}, m}^{z, \xi \eta}={ }_{n^{\prime}-1} & H_{i^{\prime}, m}^{z, \xi \eta}-\frac{\Delta t}{\mu \Delta x} \\
& \times\left\{\left({ }_{n} E_{i, m}^{y, \phi \eta}-{ }_{n} E_{i+1, m}^{y, \phi \eta}\right) \times 2^{r_{x} / 2} \delta_{p_{x}, 2^{r_{x}}-1}\right. \\
& +\sum_{r_{x}^{\prime}, p_{x}^{\prime}} \mathcal{D}_{0}\left(r_{x}, p_{x}, r_{x}^{\prime}, p_{x}^{\prime}\right)_{n} E_{i+1, m}^{y, \psi_{r_{x}^{\prime}, p_{x}^{\prime}} \eta} \\
& \left.+\sum_{r_{x}^{\prime}, p_{x}^{\prime}} \mathcal{D}_{1}\left(r_{x}, p_{x}, r_{x}^{\prime}, p_{x}^{\prime}\right)_{n} E_{i, m}^{y, \psi_{r_{x}^{\prime}, p_{x}^{\prime}} \eta}\right\}
\end{aligned}
$$

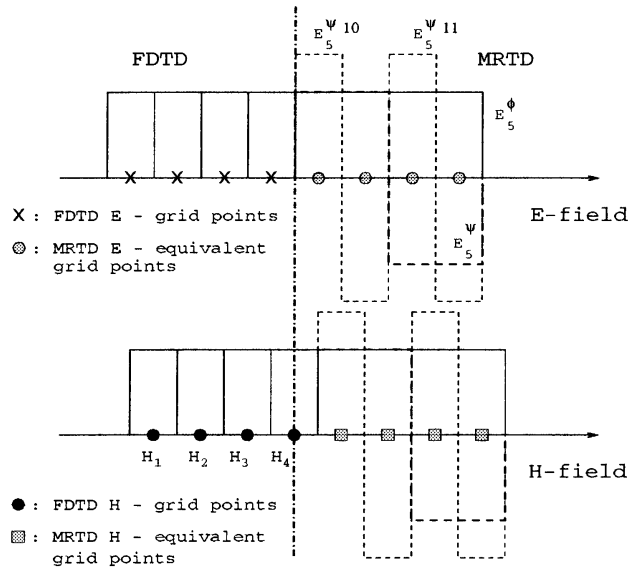

Fig. 3. One-dimensional interface between FDTD and a first-order Haar MRTD scheme.

where the coefficients $\mathcal{D}_{0}$ and $\mathcal{D}_{1}$ are given in Appendix, $i^{\prime}=$ $i+s_{x}, m^{\prime}=m+s_{z}, n^{\prime}=n+1 / 2, \xi=\psi_{r_{x}, p_{x}}$, and $\zeta=\psi_{r_{z}, p_{z}}$, while $\chi=\phi, \psi_{r_{x}, p_{x}}$ and $\eta=\phi, \psi_{r_{z}, p_{z}}$. The update equations for $E_{y}$ assume a similar form and involve the coefficients $\mathcal{D}_{2}$ and $\mathcal{D}_{3}$ of the Appendix. In addition, the well-known FDTD update equations [15] are applied in the FDTD region, which is discretized in two-dimensional Yee's cells of dimensions $\Delta x_{\text {eff }} \times \Delta z_{\text {eff }}$.

An MRTD dispersion analysis [14] leads to the following expression:

$$
\begin{aligned}
\left\{\frac{1}{u_{p} \Delta t} \sin \frac{\omega \Delta t}{2}\right\}^{2}=\left\{\frac{1}{\Delta x_{\mathrm{eff}}}\right. & \left.\sin \frac{k_{x} \Delta x_{\mathrm{eff}}}{2}\right\}^{2} \\
+ & \left\{\frac{1}{\Delta z_{\mathrm{eff}}} \sin \frac{k_{z} \Delta z_{\mathrm{eff}}}{2}\right\}^{2}
\end{aligned}
$$

which coincides with the dispersion relationship of an FDTD scheme with cell size $\Delta x_{\text {eff }} \times \Delta z_{\text {eff }}$, as the one considered here ( $u_{p}$ denotes the phase velocity). Moreover, the corresponding Courant stability condition for MRTD assumes the form

$$
\Delta t \leq \frac{1}{u_{p} \sqrt{\frac{1}{\Delta x_{\mathrm{eff}}^{2}}+\frac{1}{\Delta z_{\mathrm{eff}}^{2}}}} .
$$

Hence, under the electric/magnetic-node arrangement that is used for the MRTD region, a perfect matching of the stability and dispersion properties of the methods applied in the two regions is attained, leading to their reflectionless and stable connection with no need for any interpolation or extrapolation in either space or time.

\section{B. Connection Algorithm}

A simple one-dimensional example of an interface between a first-order Haar MRTD and the corresponding FDTD scheme is shown in Fig. 3. For the update of the electric-field MRTD coefficients $E_{5}^{\phi}, E_{5}^{\psi}, E_{5}^{\psi_{10}}$, and $E_{5}^{\psi_{11}}$, magnetic scaling, and wavelet terms one cell to the left within the FDTD region are necessary. For their calculation, the FDTD nodal values of the magnetic field $H_{1}, H_{2}, H_{3}$, and $H_{4}$ are used as an input to a recursive fast 

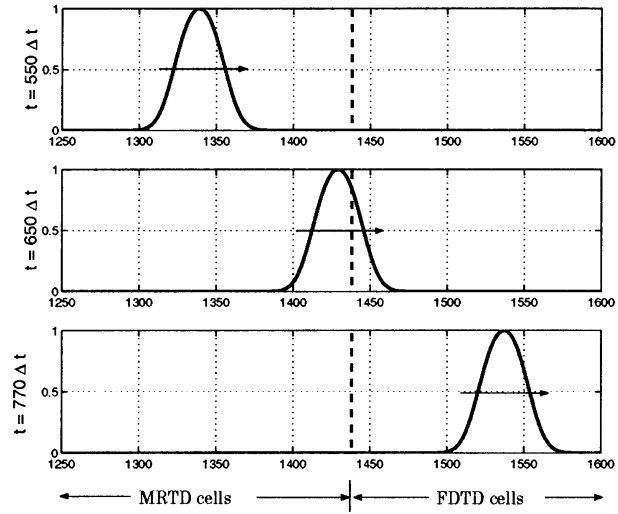

Fig. 4. Reflectionless propagation through a one-dimensional FDTD/fourthorder MRTD interface.

wavelet transform (FWT). Thus, the wavelet decomposition of the magnetic field at that cell is deduced and employed for the electric-field updates of MRTD at the FDTD/MRTD boundary. In the case of an FDTD to MRTD transition, an inverse fast wavelet transform (IFWT) is applied to derive FDTD coefficients (nodal field values) from MRTD terms. It is noted that both the FWT and IFWT are characterized by an optimal complexity of $\mathcal{O}(N)$. In addition, their computational implementation is relatively simple.

As a computational validation of these interface principles, the propagation of a $0-5-\mathrm{GHz}$ Gaussian pulse through an FDTD/fourth-order MRTD interface is shown in Fig. 4. The cell size for the FDTD region (and effective cell size for MRTD) is $2.4 \mathrm{~mm}$, while the MRTD scaling cell size is $76.8 \mathrm{~mm}$. The time step is 0.9 of the Courant limit. The interface for the electric-field nodes is located at cell 1440. Smooth and stable transition from the MRTD to FDTD region is observed, as expected.

Next, a two-dimensional FDTD/MRTD connection algorithm is presented for the case where an FDTD region encloses an MRTD one. An application of interest for this case is the termination of an MRTD mesh in an FDTD PML absorber, which allows for MRTD domain truncation via existing absorber codes. Similar concepts can be employed for interfaces of other types.

To begin, data transfer from the MRTD to FDTD is addressed. For the update of all FDTD grid points whose stencil extends into the MRTD region, it suffices to retrieve the nodal field values of the electric field one FDTD cell within the MRTD region (Fig. 5). Thus, the complete determination of the tangential electromagnetic-field components across the boundary of the FDTD domain is allowed for. The independent solution of this region then becomes possible since the MRTD calculated tangential fields are used as boundary conditions for FDTD. In consequence, the problem is reduced to calculating nodal field values along the boundary of the MRTD region, which is exactly the function of the IFWT (see [16]) that is carried out at the optimal complexity of $\mathcal{O}(N)$.

Conversely, for the update of the MRTD boundary magnetic-field coefficients, FDTD electric-field nodes extending over one scaling cell within the FDTD region are wavelet transformed via a FWT routine. If this scaling cell extends

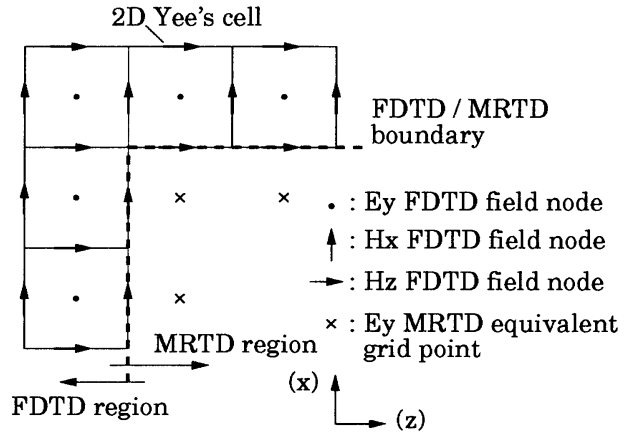

Fig. 5. FDTD update from MRTD data.

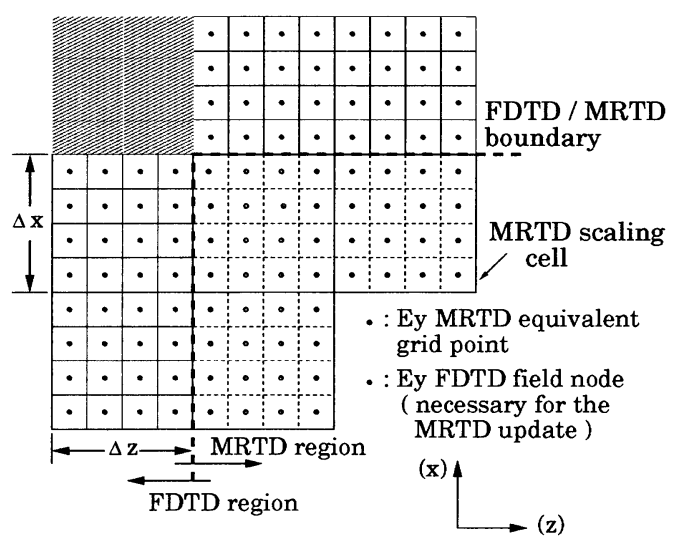

Fig. 6. MRTD update from FDTD data.

beyond the domain, zero field values are used. This is possible and physically correct for both closed- and open-domain problems, provided that a perfect conductor backed absorber is typically used for the simulation of an open boundary. Fig. 6 schematically explains this procedure for a case where $r_{x, \max }=r_{z, \max }=1$. It is noted that no FDTD grid points are sought for at the (shaded) corner region shown in Fig. 6. This is due to the fact that MRTD, just as FDTD, uses a cross-shaped stencil for the update of all grid points. Computing the electric-field MRTD coefficients from the FDTD data via an FWT and updating the tangential magnetic-field component coefficients via the standard MRTD finite-difference equations determines, again, the tangential electromagnetic-field components across the boundary of the MRTD region. This, in turn, is sufficient for its independent MRTD-based solution.

Evidently, all operations that implement this connection algorithm are performed at the same time step, during the update of the electric-field coefficients in both regions, in an absolutely stable fashion, due to the matching of the dispersion properties of the two schemes. Furthermore, the same principles lead to interfaces between wavelet schemes of an arbitrary basis and the ones that are formulated by the corresponding scaling functions only, provided that the effective resolutions in the two regions are kept the same. It is also noted that the extension of the interface algorithm to three dimensions is accomplished by treating each face of Yee's cell according to the method that has been set forth in this study. 


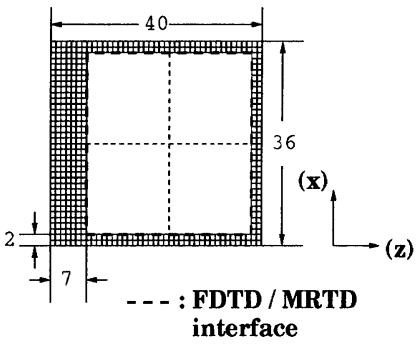

Fig. 7. Empty rectangular-cavity geometry and interface of a $3 \times 3$ order MRTD/FDTD mesh configuration (dimensions are given in FDTD cells, MRTD mesh is $2 \times 2$ ).

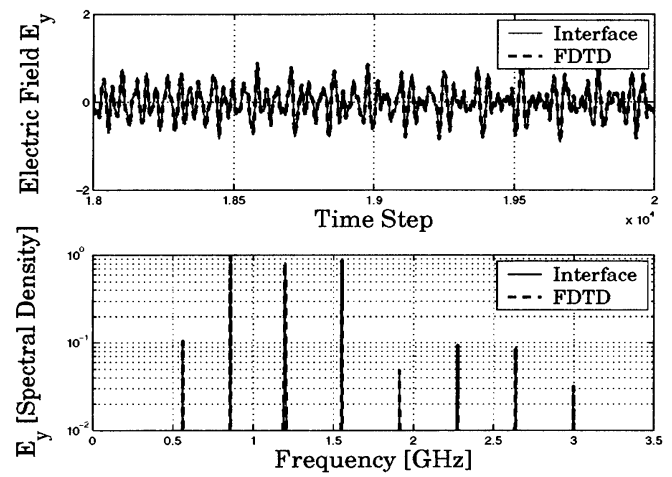

Fig. 8. Time- and frequency-domain patterns of electric field $\left(E_{y}\right)$ sampled within the cavity of Fig. 7, as obtained by the FDTD and hybrid scheme.

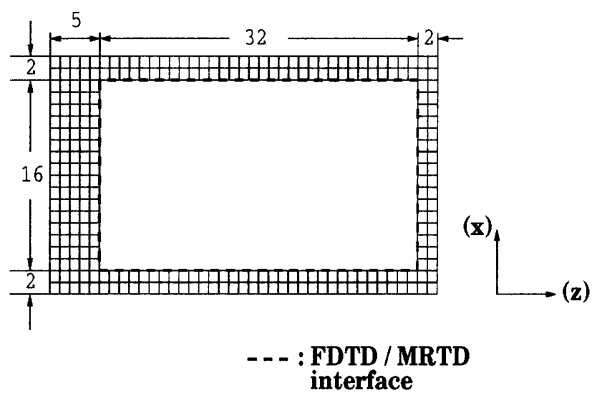

Fig. 9. Empty rectangular-cavity geometry and interface of a $3 \times 4$ order MRTD/FDTD mesh configuration (dimensions are given in FDTD cells, MRTD mesh is $1 \times 1)$.

\section{NUMERICAL RESULTS: VALIDATION}

Several two-dimensional air-filled square resonators have been analyzed in various FDTD/MRTD mesh configurations for validation purposes. The choice of those configurations was made in order to demonstrate that the stability and accuracy of the algorithm was preserved under any gridding conditions, these being either related to the order of the MRTD scheme or the proximity of the MRTD region to the hard boundary of the domain. The dimensions of the cavity structures in all figures are given in FDTD cells (or MRTD equivalent grid points). Figs. 7-10 depict two case studies for MRTD/FDTD mesh configurations. In the first case (Fig. 7), an MRTD scheme of order 3 in both the $x$ - and $z$-direction forms a mesh of $2 \times 2$ scaling cells corresponding to $32 \times 32$ FDTD cells, asymmetrically placed within an FDTD region that is terminated into the metal boundaries of the cavity. The whole domain corresponds to

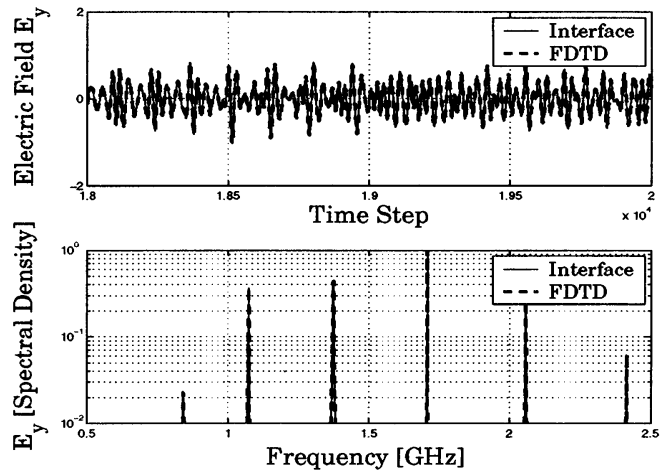

Fig. 10. Time- and frequency-domain patterns of electric field $\left(E_{y}\right)$ sampled within the cavity of Fig. 9, as obtained by the FDTD and hybrid scheme.

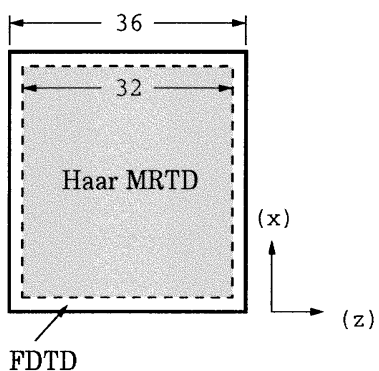

Fig. 11. Empty square-cavity geometry and interface of a $4 \times 4$ order MRTD/FDTD mesh configuration (dimensions are given in FDTD cells, MRTD mesh is $1 \times 1$ )

$36 \times 40$ FDTD cells of dimension $1 \mathrm{~cm} \times 1 \mathrm{~cm}$. A pure MRTD scheme would model these electric walls by means of image theory, necessitating the introduction of several images of high-order wavelet terms [5]. On the other hand, enclosing the MRTD region in an FDTD one facilitates the treatment of these hard boundaries, whose FDTD modeling amounts to setting the tangential to perfect electric conducting (PEC) electric field nodal values equal to zero. Similarly, in the second case, an MRTD scheme of orders 3 and 4 in the $x$ - and $z$-direction, respectively, forms a $1 \times 1$ scaling mesh and is interfaced with FDTD of cell size $1 \mathrm{~cm} \times 1 \mathrm{~cm}$. The total domain corresponds to $20 \times 39$ FDTD cells. Cavity resonances that are derived via a pure FDTD scheme and the proposed interface-based method agree well (in both the time and frequency domains), as shown in Figs. 8 and 10. In all cases, the time step was set equal to 0.9 of the Courant stability limit.

A third validation case is shown in Fig. 11. In that case, the MRTD region is symmetrically placed within the FDTD mesh and only two FDTD cells away from the boundary. The MRTD scheme is of order 4 in both directions, hence, giving rise to a single scaling cell mesh in the MRTD region. The effective cell size is again $1 \mathrm{~cm} \times 1 \mathrm{~cm}$ and the time step equal to 0.9 of the Courant limit. The patterns of the $\mathrm{TE}_{21}$ and $\mathrm{TE}_{22}$ modes, derived via the interface algorithm, are shown in Figs. 12 and 13. The smooth patterns confirm the correctness of the scheme and the absence of any type of spurious effects that would corrupt its performance. This observation is also in agreement with the Haar MRTD dispersion analysis in [14]. 


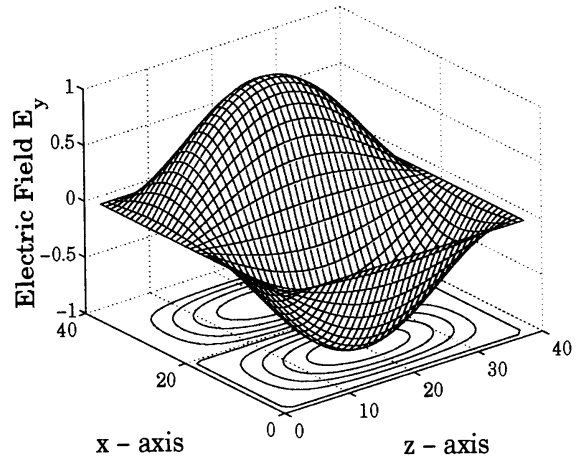

Fig. 12. Electric-field pattern for the $\mathrm{TE}_{21}$ mode of the empty cavity of Fig. 8 $(4 \times 4$ MRTD $)$.

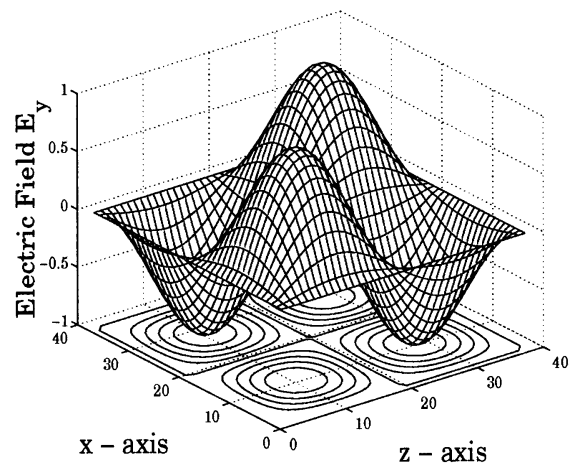

Fig. 13. Electric-field pattern for the $\mathrm{TE}_{22}$ mode of the empty cavity of Fig. 8 $(4 \times 4$ MRTD $)$.

\section{NUMERICAL RESULTS: APPLICATIONS}

\section{A. Metal Fin Loaded Cavity}

The method of this paper is applied for the simulation of a metal fin loaded cavity, similar to the one presented in [11]. This structure is chosen for the reason that the presence of the metal fin within the domain restricts the order of the MRTD scheme that can be employed for its analysis. In particular, whenever a scaling cell greater than the fin dimensions is chosen, the utmost care is necessary for the compensation of the unphysical coupling of the regions below and above the fin, which is caused by the scaling function defining the fin cell (or wavelets extending beyond the fin limits). However, the strategies that are followed in this case (e.g., domain split [17]) result in a local increase of operations and consumption of computational resources and bring about practical implementation problems, especially when one is interested in developing generic wavelet-based computeraided design (CAD) tools.

For the interface-based solution of the problem, the gridding conditions are given in Fig. 14. In the MRTD region, a second by second-order scheme is employed $(4 \times 4$ scaling cells). The time step is set at 0.8 of the Courant limit and FDTD cells of $1 \mathrm{~cm} \times 1 \mathrm{~cm}$ are used. As an excitation, a Gaussian pulse, with its 3-dB frequency chosen to be equal to $f_{c} / 2, f_{c}$ being the cutoff frequency of the $\mathrm{TE}_{11}$ mode of the cavity, is applied in the FDTD region (at the plane $z=5 \mathrm{~cm}$ ). Under these conditions, a stable performance of the code was obtained. The deduced electric-field spatial distribution is shown in Fig. 15. Moreover, in order to demonstrate the stability of the solution,

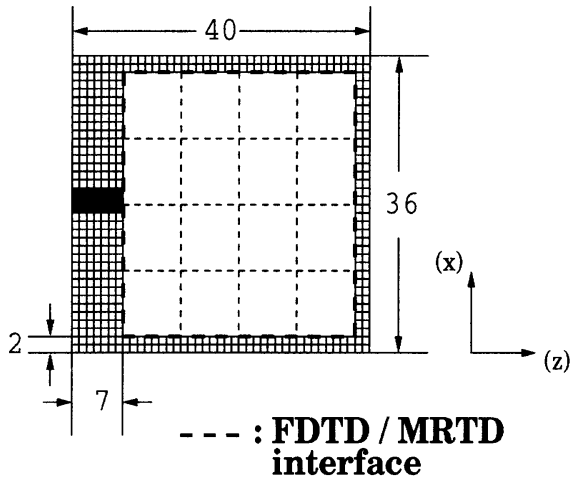

Fig. 14. Metal fin loaded cavity geometry and interface of a $2 \times 2$ order MRTD/FDTD mesh configuration (dimensions are given in FDTD cells, MRTD mesh is $4 \times 4$ ).

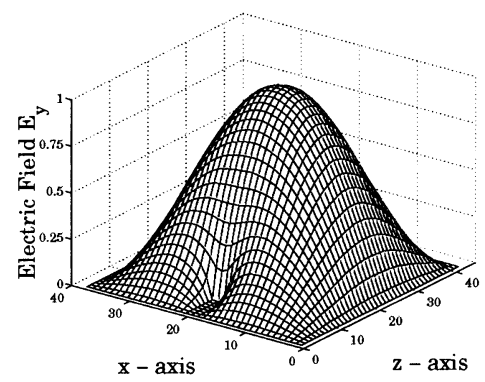

Fig. 15. Electric-field distribution in the metal fin loaded cavity (dominant TE mode).

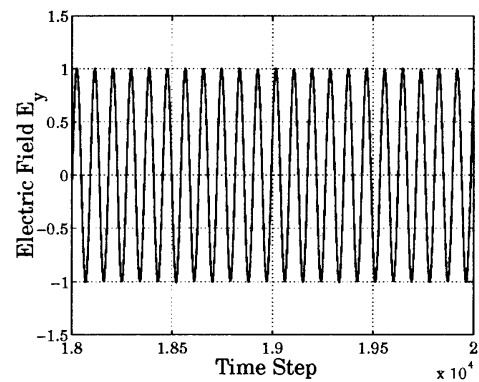

Fig. 16. Electric-field sampled within the fin loaded cavity as a function of time for time steps $18000-20000$.

the electric field, sampled at the point $(x=1.55 \mathrm{~cm}, z=$ $2.35 \mathrm{~cm}$ ), is plotted as a function of time for an arbitrary interval of 18 000-20 000 time steps, as shown in Fig. 16. It is noted that no late-time instabilities were observed over as many as 100000 time steps.

\section{B. PML Mesh Termination for MRTD}

A typical problem related to higher order and multiresolution formulations of finite-difference schemes is the inherently complex modeling of boundary and material conditions. For high-order Haar wavelet schemes, a formulation for a matchedlayer absorber that was proposed in [18] highlighted the difficulty of such efforts that essentially stems from the sampling of varying electric and magnetic conductivities by a multilevel basis. Furthermore, it may seem necessary that the absorber region of an MRTD mesh be discretized by at least the degrees of freedom of a single MRTD cell. However, for highorder schemes, this would lead to absorber regions that are much 


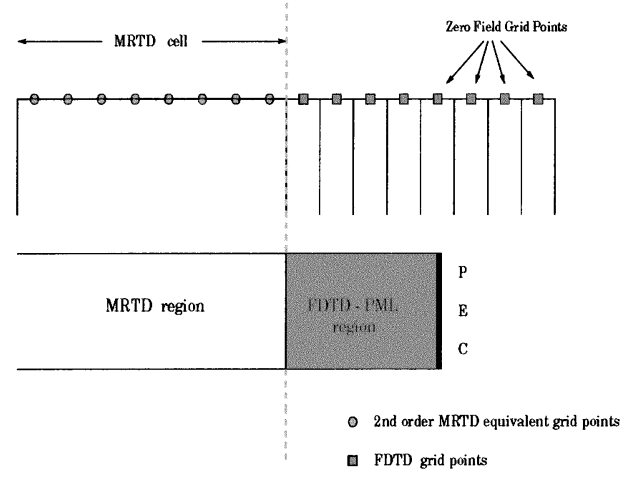

Fig. 17. Concept of MRTD mesh termination via an FDTD/MRTD interface.

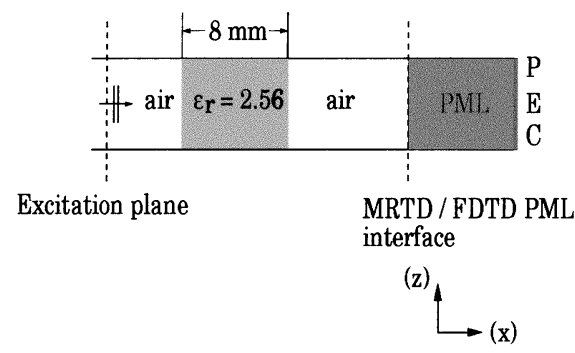

Fig. 18. Slab waveguide geometry.

longer than the ones typically used in FDTD applications. For example, terminating an MRTD with five wavelet levels (fourthorder scheme) in just a single cell of it is equivalent to using an FDTD absorber of 64 grid points per direction. Nevertheless, excellent performance of just 8-16 cell optimized FDTD-uniaxial perfectly matched layer (UPML) absorbers has been recently demonstrated [19], prompting the quest for MRTD absorbers that may extend over a fraction of an MRTD scaling cell.

In this paper, the concept of the FDTD/MRTD interface is employed for the implementation of a UPML termination of an MRTD domain. In particular, an FDTD-UPML region encloses an MRTD one and the interface algorithm is applied for the reflectionless connection of the two. In case the FDTD region corresponds to a fraction of a scaling cell, the application of MRTD update equations needs FDTD grid points beyond the conductor that backs the PML. The latter are simply zeroed out and fed back as such to the FWT routine. This concept is explained for a one-dimensional case of a second-order MRTD scheme (with eight equivalent grid points per cell) terminated into a four-cell FDTD PML (half a scaling cell), as shown in Fig. 17.

Using this method, the waveguide structure of Fig. 18, also presented in [6] and [7], is solved by a fourth-order MRTD scheme, truncated with a six- and eight-grid-point PML corresponding to 0.1875 and 0.25 of a scaling cell, which, in this case, is $8 \mathrm{~mm}$. A $0-30-\mathrm{GHz}$ Gaussian pulse excitation is used, and the reflection coefficient from the slab is calculated. The scaling cell is $1.28 \times \lambda_{\min }$, while the five wavelet levels successively refine the resolution of the scheme to $\lambda_{\min } / 25$. An important principle of multiresolution analysis is thus demonstrated. Loss of accuracy and aliasing due to the violation of the Nyquist limit at the scaling function level is recursively compensated for by the higher order wavelets. Fig. 19 depicts comparative plots

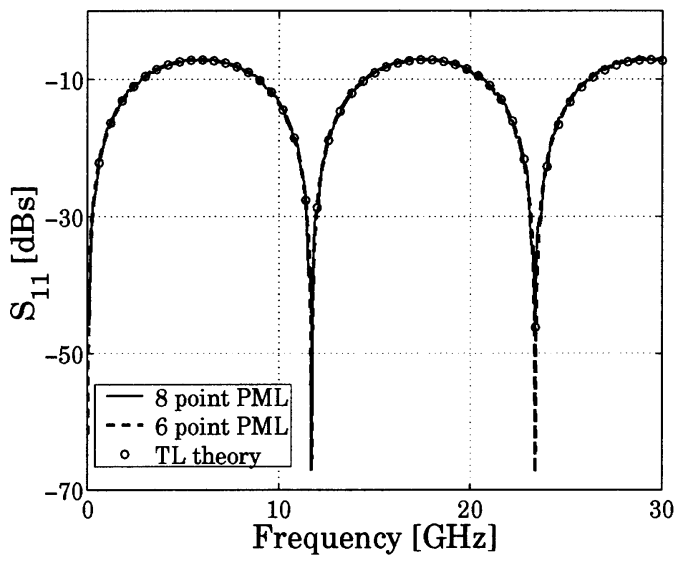

Fig. 19. Numerical and theoretical $S_{11}$ for the slab geometry.

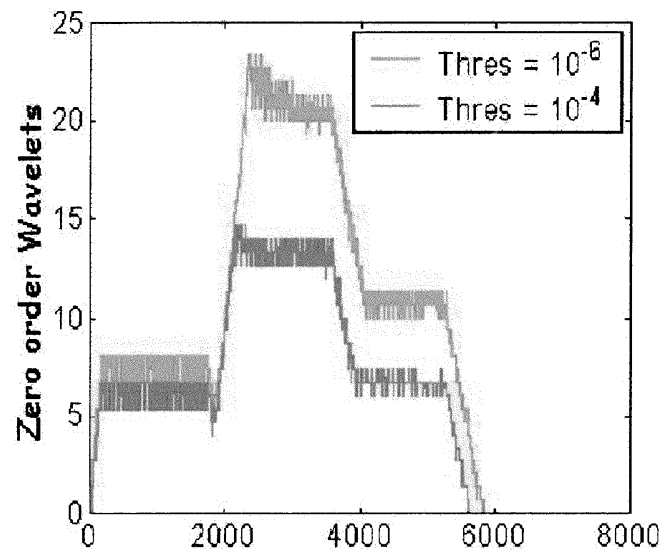

Fig. 20. Unthresholded to the total number (in percentage) of zeroth-order wavelet coefficients at each time step for a fourth-order Haar MRTD simulation of the problem of Fig. 18.

of the numerical results derived by the two termination types, along with the theoretical $S_{11}$ form derived by transmission-line theory. Evidently, all three sets of results are in good correlation with each other.

Thresholding of Haar wavelet coefficients is applied based on the condition

$$
\left|n E_{j}^{\psi_{r, p}}\right| \leq 2^{-r / 2} \epsilon
$$

where $\epsilon$ is a predefined threshold. Hence, the amplitude of the threshold is adapted to the order $r$ of each wavelet coefficient through the use of a normalization term $2^{-r / 2}$. This choice is due to the fact that the maximum absolute value of the basis function $\psi_{j, p}^{r}(x)$ is $2^{r / 2}$. Thresholding then amounts to omitting wavelet terms whose maximum contribution to the field value is less than $\epsilon$. For two different threshold values $\epsilon=10^{-6}$ and $10^{-4}$, the number of wavelet coefficients that are above threshold is computed at each time step. The resultant plots for wavelets of orders 0-3 are shown in Figs. 20-23. Evidently, despite small differences between them, all four curves have a similar pattern: Around time-step 2000, unthresholded wavelet coefficients are doubled as a result of the pulse incidence on the dielectric slab, which generates an additional (reflected) wavefront. The PML absorption of the two wavefronts, symmetrically generated by the source in the middle of the domain, then 


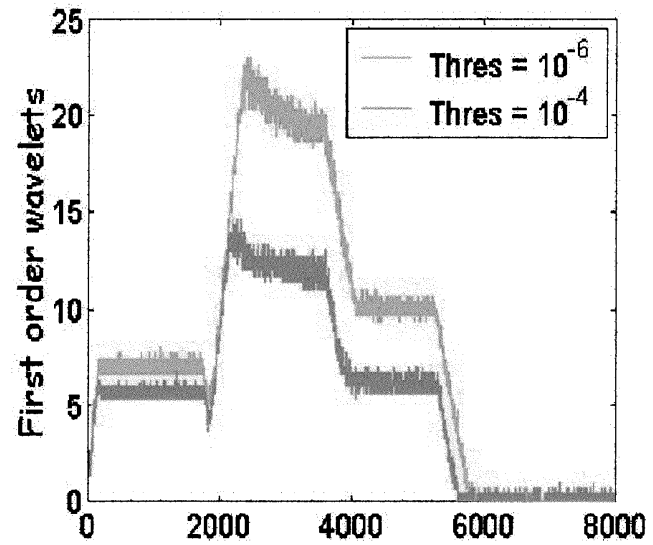

Fig. 21. Unthresholded to total number (in percentage) of first-order wavelet coefficients at each time step for a fourth-order Haar MRTD simulation of the problem of Fig. 18.

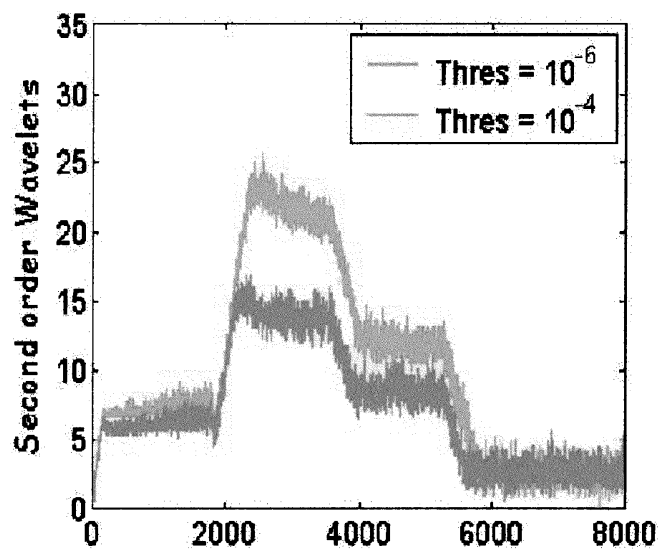

Fig. 22. Unthresholded to total number (in percentage) of second-order wavelet coefficients for a fourth-order Haar MRTD simulation of the problem of Fig. 18.

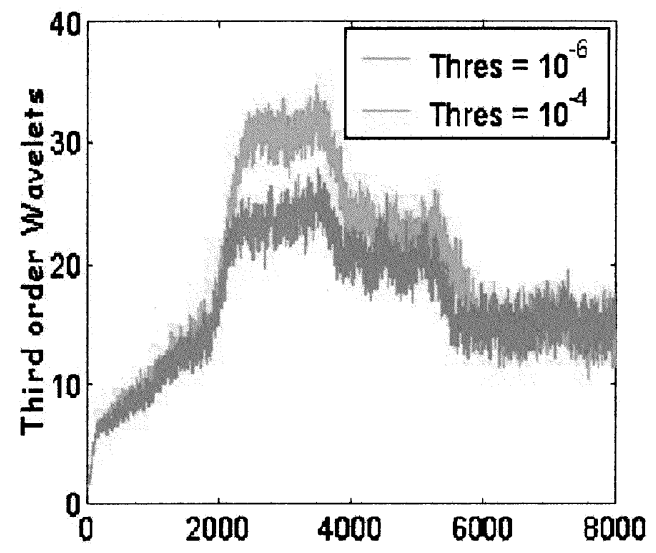

Fig. 23. Unthresholded to total number (in percentage) of third-order wavelet coefficients, at each time step for a fourth-order Haar MRTD simulation of the problem of Fig. 18.

causes a step-wise decrease in the number of active wavelet coefficients. Finally, the absorption of the reflected wavefront signals the end of the simulation and the decay of the number of unthresholded coefficients to almost zero. Overall, thresholding of wavelet coefficients yields a compression in memory requirements by $64.6 \%$ (in 8192 time steps). It is also noted that, the

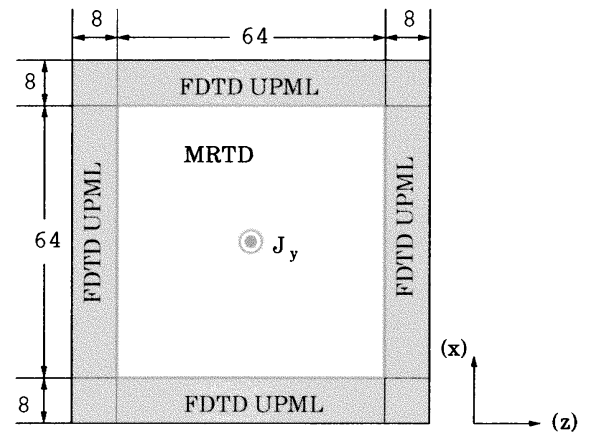

Fig. 24. Case study for the interface-based termination of a two-dimensional MRTD domain.

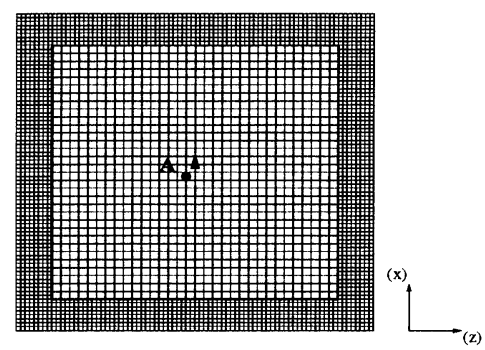

Fig. 25. FDTD UPML interfaced with a $0 \times 0$ order MRTD (MRTD mesh is $32 \times 32$ ).

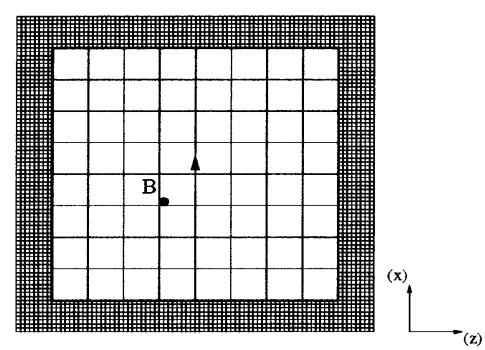

Fig. 26. FDTD-UPML interfaced with a $2 \times 2$ order MRTD (MRTD mesh is $8 \times 8)$.

higher the order of wavelet terms, the higher the number of steady-state unthresholded wavelets (that theoretically should be zero). This stems from the fact that high-order wavelets are excited by numerical errors due to the finite discretization of the domain and absorber. This observation presents an ultimate limit for the efficiency of wavelet methods, but also underlines the importance of MRTD mesh termination studies. Obviously, inefficient absorbers produce significant reflection errors that excite high-order wavelet terms, keeping them above threshold even at late simulation times.

Moreover, a two-dimensional eight-cell UPML with theoretical reflection coefficient $R=\exp (-16)$ and fourth-order polynomial conductivity variation is used to terminate MRTD meshes that correspond to a $64 \times 64$ FDTD domain (Fig. 24). The three case studies are depicted in Figs. 25-27 and correspond to MRTD orders $0 \times 0,2 \times 2$, and $4 \times 4$, respectively. An electric-current excitation of the form

$$
J_{y}(t)=\frac{1}{\Delta x \Delta z}\left(4\left(\frac{t}{t_{0}}\right)^{3}-\left(\frac{t}{t_{0}}\right)^{4}\right) \exp \left(\frac{-t}{t_{0}}\right)
$$




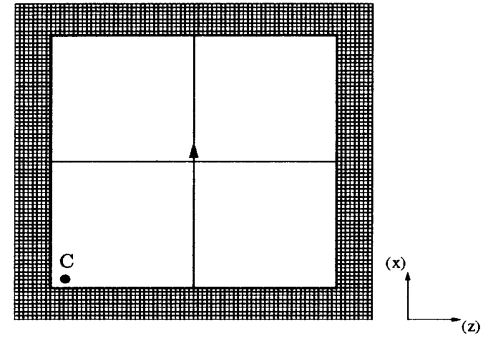

Fig. 27. FDTD-UPML interfaced with a $4 \times 4$ order MRTD (MRTD mesh is $2 \times 2)$.

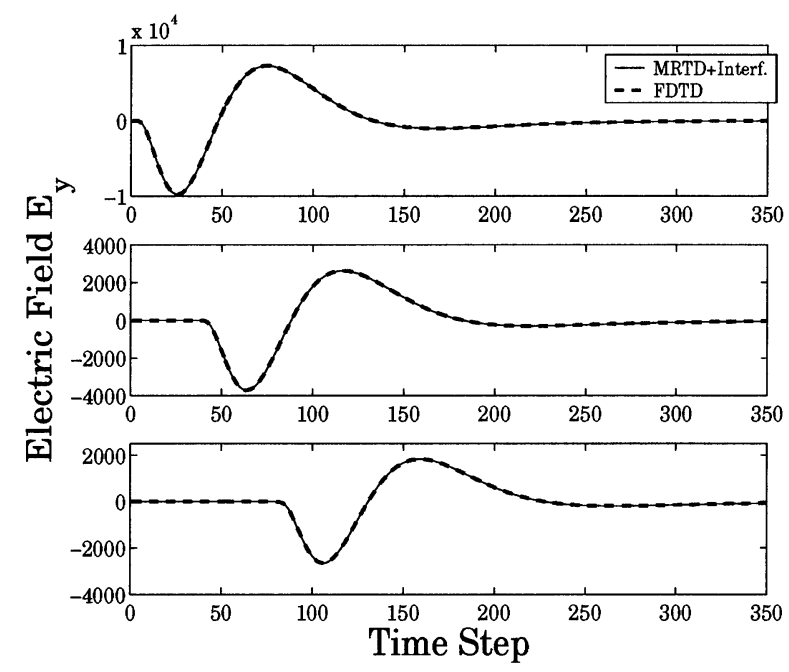

Fig. 28. Comparison of FDTD and MRTD/FDTD interface results for the three case studies of Figs. 25-27.

with $t_{0}=1 /\left(4 \pi f_{0}\right)$ and $f_{0}=1 \mathrm{GHz}$ is applied in the middle of the domain. The FDTD cell size is set equal to $2.5 \mathrm{~mm} \times 2.5 \mathrm{~mm}$ and the time step is $\Delta t=4.5 \mathrm{ps}$. Time-domain field waveforms are then sampled at the points indicated as $A, B, C$ in Figs. 25-27, corresponding to FDTD cells $(31,31),(17,17)$, and $(1,1)$ of the $64 \times 64$ domain. The results, shown in Fig. 28, demonstrate an excellent agreement between the pure FDTD scheme and the FDTD-UPML terminated MRTD. It is noted that the UPML regions in these three examples extend over 4, 1 , and 0.25 MRTD cells, respectively, demonstrating the ability of the interface to provide MRTD absorbing boundary conditions (ABCs) with efficiency and complexity that are independent of the order of the underlying wavelet expansion.

In addition, Fig. 29 depicts the electric-field waveform sampled at point $B$ of the MRTD domain of Fig. 26 when terminated at 4,8 , and 16 FDTD-UPML cells $(0.5,1$, and 2 MRTD cells, respectively). The same waveform is determined via the FDTD method, applied at a $64 \times 64$ mesh, terminated at 32 UPML cells. Evidently, all four curves agree well. Moreover, the broad time window over which the results are given shows the absence of any significant retro-reflections from the absorbers in all three termination schemes.

\section{Mur's ABCs for MRTD}

The application of simple ABCs assumes significant complexity in the context of multiresolution techniques, especially when arbitrarily high-order schemes are considered. The reason

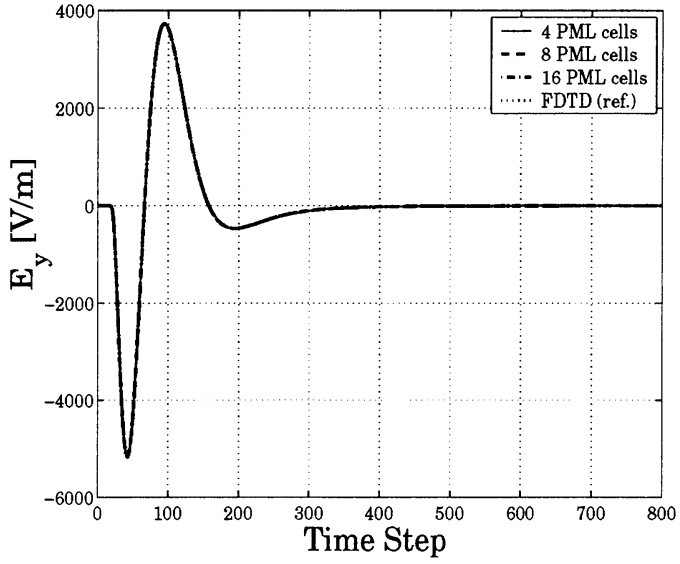

Fig. 29. Electric field at point $B$ of Fig. 26 when a 4, 8, and 16 FDTD UPML is used to terminate the MRTD mesh. An FDTD solution (with a 32-cell UPML) is appended for comparison.

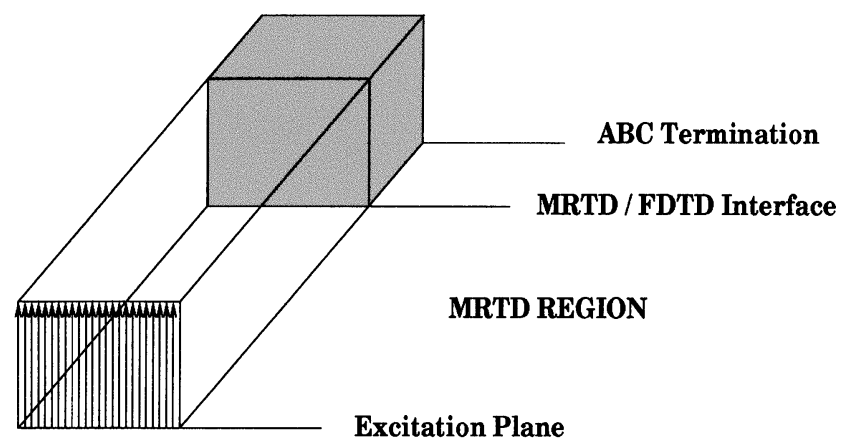

Fig. 30. Concept of ABC termination of an MRTD mesh. The FDTD region terminated into the $\mathrm{ABC}$ should at least extend over one scaling MRTD cell.

for that is that $\mathrm{ABCs}$ impose mathematical conditions at planes that include only a fraction of the equivalent grid points contained within a terminal cell. The concept of an interface-based solution to this question is depicted in Fig. 30. For the purpose of applying an ABC, an FDTD region-no shorter than a single MRTD scaling cell-is introduced. The FDTD region is then terminated into a classically implemented Mur's first-order $\mathrm{ABC}[20]$. Thus, while the boundary condition itself takes a single cell, the FDTD region now needs to extend over the degrees of freedom of an MRTD scaling cell-not a fraction of it, as in the case of a PEC-backed PML absorber. Due to this tradeoff, this mesh truncation method is more suitable to loworder MRTD schemes.

Results from a one-dimensional implementation of these concepts are shown in Figs. 31 and 32. A 0-5-GHz Gaussian pulse propagation in a TEM waveguide mode is considered. Four Haar MRTD schemes of orders $0-3$ with an effective cell size of $0.0024 \mathrm{~m}$ and time step equal to 0.9 of their Courant limit are terminated into a Mur's first-order ABC. The FDTD region occupies two MRTD scaling cells for each scheme. Therefore, the thickness of the FDTD region for each case study is 0.0096, $0.0192,0.0384$, and $0.0768 \mathrm{~m}$, respectively. That is also the reason for the phase difference of the reflected waveforms in Fig. 31. Finally, Fig. 32 presents the reflection coefficient of each of the four terminations. Obviously, the obtained $\mathrm{ABC}$ performance is similar in all MRTD cases and confirms the ca- 

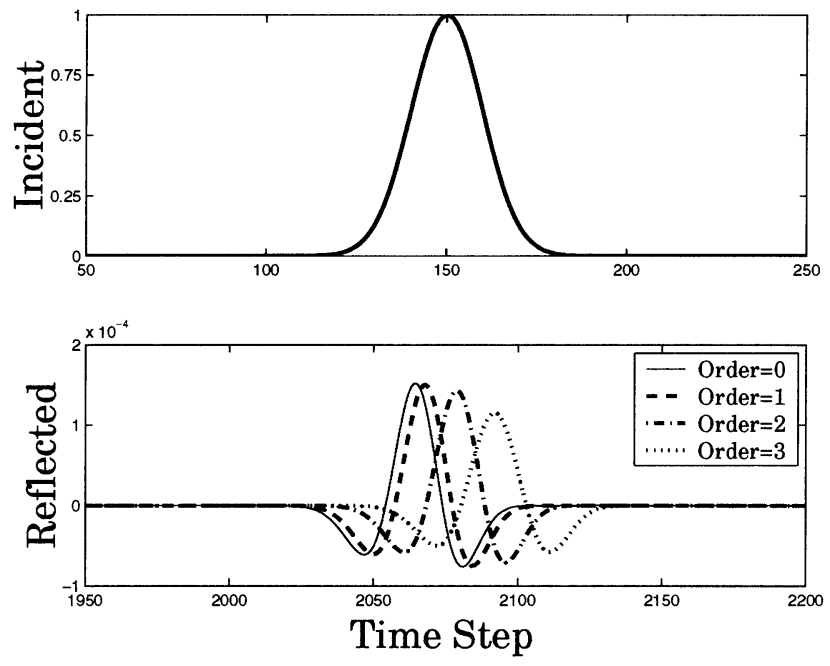

Fig. 31. Incident and reflected field waveforms for Mur's first-order $A B C$ (implemented via an FDTD/MRTD interface) in the case of one-dimensional wave propagation (Fig. 30) for MRTD orders 0-3.

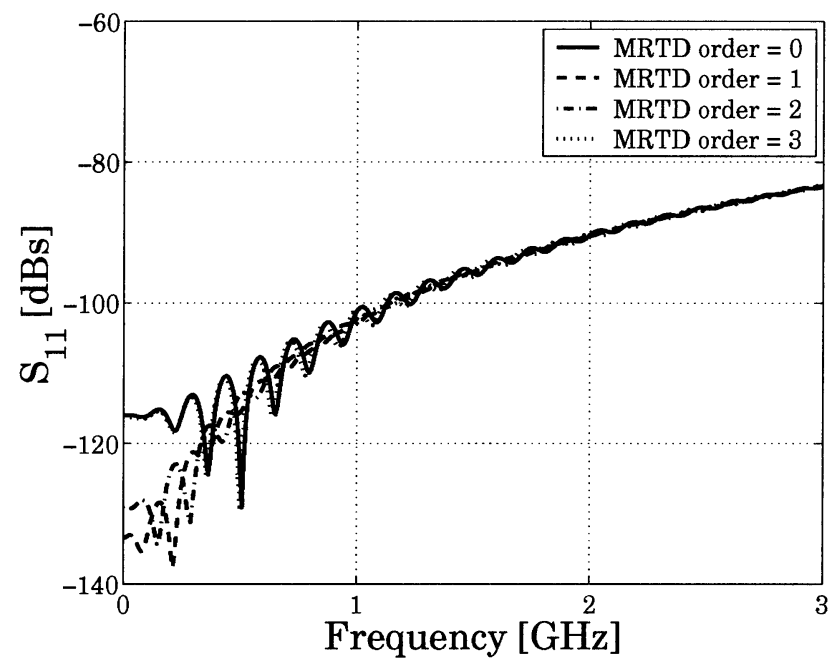

Fig. 32. Reflection coefficient for Mur's first-order ABC (implemented via an FDTD/MRTD interface) in the case of one-dimensional wave propagation (Fig. 30).

pability of the method to provide an efficient means of implementing an $\mathrm{ABC}$ truncation of wavelet grids.

\section{CONCLUSIONS}

A numerical interface between an arbitrary-order Haar MRTD scheme and the well-known FDTD technique has been demonstrated. The two unique features of the proposed method are that, first, it does not employ any interpolations or extrapolations and, second, that it applies to arbitrary-order MRTD schemes. An important application of this hybrid technique is the straightforward termination of MRTD meshes via existing FDTD-oriented PML and ABC techniques. Hence, the developed algorithm constitutes a computationally efficient tool for jointly exploiting the advantages of FDTD and MRTD, which is critical for the acceleration of time-domain numerical schemes, when applied to large-scale problems of current microwave technology structures.

\section{APPENDIX}

\section{INTEGRALS PERTINENT TO THE MoM DERIVATION OF ARBITRARY-ORDER HAAR MRTD SCHEMES}

Analytical expressions of integrals that are encountered in the application of the MoM for the derivation of Haar MRTD update equations are provided below. The maximum wavelet order that determines the mutual shift of electric-/magnetic-field nodes in axial directions is denoted as $R$, while $s_{R}=1 / 2^{R+2}$ is the shift itself as follows:

$$
\begin{aligned}
\int_{-\infty}^{+\infty} \frac{d \phi_{k^{\prime}}}{d t}(t) \phi_{k+s_{R}}(t) d t= & \delta_{k^{\prime}, k+1}-\delta_{k^{\prime}, k} \\
\int_{-\infty}^{+\infty} \frac{d \psi_{k^{\prime}, p}^{r}}{d t}(t) \phi_{k+s_{R}}(t) d t= & 2^{r / 2}\left(\delta_{k^{\prime}, k+1}-\delta_{k^{\prime}, k}\right) \delta_{p, 0} \\
\int_{-\infty}^{+\infty} \frac{d \phi_{k^{\prime}}}{d t}(t) \psi_{k+s_{R}, p}^{r}(t) d t= & 2^{r / 2}\left(\delta_{k^{\prime}, k}-\delta_{k^{\prime}, k+1}\right) \\
& \times \delta_{p, 2^{r}-1} \\
\int_{-\infty}^{+\infty} \frac{d \psi_{k^{\prime}, p^{\prime}}^{r^{\prime}}(t) \psi_{k+s_{R}, p}^{r}(t) d t=}{d t} & +\mathcal{D}_{0}\left(r, p, r^{\prime}, p^{\prime}\right) \delta_{k^{\prime}, k+1}\left(r, p, r^{\prime}, p^{\prime}\right) \delta_{k^{\prime}, k} \\
\int_{-\infty}^{+\infty} \frac{d \phi_{k^{\prime}+s_{R}}}{d t}(t) \phi_{k}(t) d t= & \delta_{k^{\prime}, k}-\delta_{k^{\prime}, k-1} \\
\int_{-\infty}^{+\infty} \frac{d \psi_{k^{\prime}+s_{R}, p}^{r}}{d t}(t) \phi_{k}(t) d t= & 2^{r / 2}\left(\delta_{k^{\prime}, k-1}-\delta_{k^{\prime}, k}\right) \\
& \times \delta_{p, 2^{r}-1} \\
\int_{-\infty}^{+\infty} \frac{d \phi_{k^{\prime}+s_{R}}}{d t}(t) \psi_{k, p}^{r}(t) d t= & 2^{r / 2}\left(\delta_{k^{\prime}, k}-\delta_{k^{\prime}, k-1}\right) \delta_{p, 0} \\
\int_{-\infty}^{+\infty} \frac{d \psi_{k^{\prime}+s_{R}, p^{\prime}}^{r^{\prime}}}{d t}(t) \psi_{k, p}^{r}(t) d t= & \mathcal{D}_{2}\left(r, p, r^{\prime}, p^{\prime}\right) \delta_{k^{\prime}, k}\left(r, p, r^{\prime}, p^{\prime}\right) \delta_{k^{\prime}, k-1} \\
& \left.+\mathcal{D}_{3}(1)\right)
\end{aligned}
$$

with

$$
\begin{aligned}
& \mathcal{D}_{0}\left(r, p, r^{\prime}, p^{\prime}\right)=2^{\left(r+r^{\prime}\right) / 2}\left\{\psi_{0}\left(\xi_{1}\right)-2 \psi_{0}\left(\xi_{2}\right)+\psi_{0}\left(\xi_{3}\right)\right\} \\
& \mathcal{D}_{1}\left(r, p, r^{\prime}, p^{\prime}\right)=2^{\left(r+r^{\prime}\right) / 2}\left\{\psi_{0}\left(\xi_{1}^{\prime}\right)-2 \psi_{0}\left(\xi_{2}^{\prime}\right)+\psi_{0}\left(\xi_{3}^{\prime}\right)\right\} \\
& \mathcal{D}_{2}\left(r, p, r^{\prime}, p^{\prime}\right)=-\mathcal{D}_{1}\left(r, 2^{r}-p-1, r^{\prime}, 2^{r^{\prime}}-p^{\prime}-1\right) \\
& \mathcal{D}_{3}\left(r, p, r^{\prime}, p^{\prime}\right)=-\mathcal{D}_{0}\left(r, 2^{r}-p-1, r^{\prime}, 2^{r^{\prime}}-p^{\prime}-1\right)
\end{aligned}
$$

and

$$
\begin{aligned}
& \xi_{1}=2^{r}\left(1+\frac{p^{\prime}}{2^{r^{\prime}}}-\frac{1}{2^{R+2}}\right)-p \\
& \xi_{2}=2^{r}\left(1+\frac{p^{\prime}+0.5}{2^{r^{\prime}}}-\frac{1}{2^{R+2}}\right)-p \\
& \xi_{3}=2^{r}\left(1+\frac{p^{\prime}+1}{2^{r^{\prime}}}-\frac{1}{2^{R+2}}\right)-p \\
& \xi_{1}^{\prime}=2^{r}\left(\frac{p^{\prime}}{2^{r^{\prime}}}-\frac{1}{2^{R+2}}\right)-p \\
& \xi_{2}^{\prime}=2^{r}\left(\frac{p^{\prime}+0.5}{2^{r^{\prime}}}-\frac{1}{2^{R+2}}\right)-p \\
& \xi_{3}^{\prime}=2^{r}\left(\frac{p^{\prime}+1}{2^{r^{\prime}}}-\frac{1}{2^{R+2}}\right)-p .
\end{aligned}
$$


Finally, $\delta_{\kappa, \lambda}$ is the well-known Kronecker delta and $\psi_{0}$ is the Haar mother wavelet function. The previous expressions are readily programmabe and allow for the development of arbitrary-order Haar wavelet MRTD codes. Yet code efficiency is greatly enhanced by a priori recognizing the nonzero coefficients $\mathcal{D}_{0}, \mathcal{D}_{1}, \mathcal{D}_{2}$, and $\mathcal{D}_{3}$ and omitting operations that involve multiplications by zero in the main time-stepping loop. This is done at the preprocessing stage of an MRTD code.

\section{ACKNOWLEDGMENT}

Author C. D. Sarris acknowledges an illuminating communication with Prof. W. J. R. Hoefer, University of Victoria, Victoria, BC, Canada, and Dr. M. Fujii, University of Victoria, on the motivation and performance of their TLM/Haar MRTD numerical interface.

\section{REFERENCES}

[1] R. Lotz and F. Arndt, "Advanced subgrid FD technique for modeling waveguide structures with curved conducting and dielectric boundaries," in Proc. 30th Eur. Microwave Conf., Paris, France, Oct. 2000, pp. $288-291$.

[2] I. S. Kim and W. J. R. Hoefer, "A local mesh refinement algorithm for the time domain finite difference method using Maxwell's Curl equations," IEEE Trans. Microwave Theory Tech., vol. 38, pp. 812-815, June 1990.

[3] M. Okoniewski, E. Okoniewska, and M. Stuchly, "Three-dimensional subgridding algorithm for FDTD," IEEE Trans. Antennas Propagat., vol. 45, pp. 422-429, Mar. 1997.

[4] J. Ritter and F. Arndt, "A generalized 3D subgrid technique for the FDTD method," in IEEE MTT-S Int. Microwave Symp. Dig., 1997, pp. $1563-1566$.

[5] M. Krumpholz and L. P. B. Katehi, "MRTD: New time domain schemes based on multiresolution analysis," IEEE Trans. Microwave Theory Tech., vol. 44, pp. 555-561, Apr. 1996.

[6] E. Tentzeris, R. Robertson, A. Cangellaris, and L. P. B. Katehi, "Spaceand time- adaptive gridding using MRTD," in IEEE MTT-S Int. Microwave Symp. Dig., 1997, pp. 337-340.

[7] C. D. Sarris and L. P. B. Katehi, "On the use of wavelets for the implementation of high order mesh refinement in time domain simulations," in Proc. 30th Eur. Microwave Conf., vol. 3, 2000, pp. 284-287.

[8] - "Development and application of an efficient FDTD/MRTD numerical interface," in IEEE MTT-S Int. Microwave Symp. Dig., vol. 2, 2001, pp. 753-756.

[9] Z. Huang, K. Demarest, and R. G. Plumb, "An FDTD/MOM hybrid technique for modeling complex antennas in the presence of heterogeneous grounds," IEEE Trans. Geosci. Remote Sensing, vol. 37, pp. 2692-2698, Nov. 1999.

[10] T. I. Kosmanis, N. V. Kantartzis, and T. D. Tsiboukis, "A hybrid FDTDwavelet-Galerkin technique for the numerical analysis of field singularities inside waveguides," IEEE Trans. Magn., vol. 36, pp. 902-906, July 2000.

[11] M. Fujii, P. P. M. So, E. Hu, W. Liu, and W. J. R. Hoefer, "A 2D TLM and Haar MRTD real time hybrid connection algorithm," in Proc. 16th Annu. Rev. Progress Applied Computer Electromagnetics, Monterey, CA, Mar. 2000, pp. 1013-1020.

[12] S. Ju, D.-H. Bae, and H. Kim, "The modeling of lumped elements using the Haar wavelet multiresolution time domain technique," in Proc. URSI Meeting, Salt Lake City, UT, 2000.

[13] M. Fujii and W. J. R. Hoefer, "A three-dimensional Haar wavelet-based multi-resolution analysis similar to the 3-D FDTD method-Derivation and application," IEEE Trans. Microwave Theory Tech., vol. 46, pp. 2463-2475, Dec. 1998.

[14] C. D. Sarris and L. P. B. Katehi, "Fundamental gridding related dispersion effects in MRTD schemes," IEEE Trans. Microwave Theory Tech., vol. 49, pp. 2248-2257, Dec. 2001.

[15] A. Taflove, Computational Electrodynamics: The Finite Difference Time Domain Method. Norwood, MA: Artech House, 1995.

[16] I. Daubechies, Ten Lectures on Wavelets. Philadelphia, PA: SIAM, 1992.
[17] E. M. Tentzeris, A. Cangellaris, L. P. B. Katehi, and J. F. Harvey, "Multiresolution time-domain (MRTD) adaptive schemes using arbitrary resolutions of wavelets," IEEE Trans. Microwave Theory Tech., vol. 50, pp. 501-516, Feb. 2002

[18] K. Goverdhanam, C. D. Sarris, M. M. Tentzeris, and L. P. B. Katehi, "A perfectly matched layer formulation for Haar wavelet based MRTD," in Proc. 29th Eur. Microwave Conf., vol. 3, 1999, pp. 243-246.

[19] S. Gedney, "The perfectly matched layer absorbing medium," in $A d$ vances in Computational Electrodynamics: The Finite Difference Time Domain Method, A. Taflove, Ed. Norwood, MA: Artech House, 1998, ch. 5 .

[20] G. Mur, "Absorbing boundary conditions for the finite-difference approximation of the time-domain electromagnetic field equations," IEEE Trans. Electromagn. Comput., vol. EMC-23, pp. 377-382, 1981.

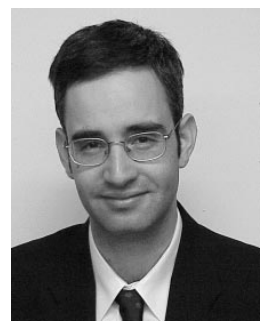

Costas D. Sarris (M'03) received the Diploma (with distinction) in electrical and computer engineering from the National Technical University of Athens (NTUA), Athens, Greece in 1997, and the M.Sc. degree in electrical engineering, M.Sc. degree in applied mathematics, and Ph.D. degree in electrical engineering from the University of Michigan at Ann Arbor, in 1998, 2002, and 2002, respectively.

In November 2002, he joined the Edward S. Rogers Sr. Department of Electrical and Computer Engineering, University of Toronto, Toronto, ON, Canada, where he is currently an Assistant Professor.

Dr. Sarris was the recipient of four Hellenic Fellowship Foundation Prizes for the academic years 1993-1997, three Technical Chamber of Greece Awards for the academic years 1994-1997 (all of them for scholastic achievement), and the 1997 NTUA/ECE Class Bronze Medal. He was also the recipient of a Student Paper Award (honorable mention) presented at the 2001 IEEE Microwave Theory and Techniques Society (IEEE MTT-S) International Microwave Symposium, Phoenix, AZ.

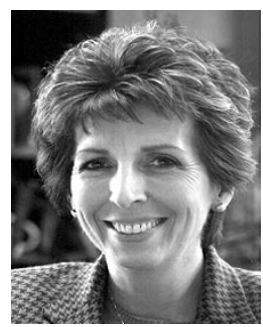

Linda P. B. Katehi (S'81-M'84-SM'89-F'95) received the B.S.E.E. degree from the National Technical University of Athens, Athens, Greece, in 1977, and the M.S.E.E. and Ph.D. degrees from the University of California at Los Angeles, in 1981 and 1984, respectively.

In September 1984, she joined the faculty of the Electrical Engineering and Computer Science Department, The University of Michigan at Ann Arbor, as an Assistant Professor, and then became an Associate Professor in 1989 and Professor in 1994. She has served in many administrative positions, including Director of Graduate Programs, College of Engineering (1995-1996), Elected Member of the College Executive Committee (1996-1998), Associate Dean For Graduate Education (1998-1999), and Associate Dean for Academic Affairs (since September 1999). She is currently the Dean of the Schools of Engineering, Purdue University, West Lafayette, IN. She has authored or coauthored 410 papers published in refereed journals and symposia proceedings and she holds four U.S. patents. She has also generated $20 \mathrm{Ph}$.D. students.

Dr. Katehi is a member of the IEEE Antennas and Propagation Society (IEEE AP-S), the IEEE Microwave Theory and Techniques Society (IEEE MTT-S), Sigma Xi, Hybrid Microelectronics, and URSI Commission D. She was a member of the IEEE AP-S AdCom (1992-1995). She was an associate editor for the IEEE TRANSACTIONS ON MICROWAVE THEORY AND TECHNIQUES and the IEEE TRansactions on ANTENNAS AND PROPagation. She was the recipient of the 1984 IEEE AP-S W. P. King (Best Paper Award for a Young Engineer), the 1985 IEEE AP-S S. A. Schelkunoff Award (Best Paper Award), the 1987 National Science Foundation Presidential Young Investigator Award, the 1987 URSI Booker Award, the 1994 Humboldt Research Award, the 1994 University of Michigan Faculty Recognition Award, the 1996 IEEE MTT-S Microwave Prize, the 1997 International Microelectronics and Packaging Society (IMAPS) Best Paper Award, and the 2000 IEEE Third Millennium Medal. 